\title{
Factors Affecting Gingivitis among Patients Attending in a Tertiary Care Hospital of Bangladesh
}

\author{
Mst. Mostary Zannath ${ }^{1}$, Md. Monoarul Haque ${ }^{2}$, Masuma Akther ${ }^{3}$
}

\begin{abstract}
Background: Gingivitis is one of the common diseases in Bangladesh and a public health problem also. Broadly factors of two categories are responsible for the occurrence of gingivitis: anatomical and behavioral. Few physiological factors also have influence. In Bangladesh we have no national health survey on periodontal condition. Objective: The aim of this study was to find out the factors affecting gingivitis among patients of 15 to 40 years of age attending in a tertiary care hospital in Dhaka city. Materials and method: This was a cross sectional study and purposive sampling method was used. Total 200 samples were taken who fulfilled the inclusion criteria by pretested structured questionnaire in outpatient department (OPD) of Bangabandhu Sheikh Mujib Medical University, Dhaka, Bangladesh. Results: The mean $( \pm S D)$ age of respondents was $27 \pm 5$ years. Dietary factors responsible for gingivitis were sweets (80\%), followed by fast food (14\%). Regarding behavioral factors, smoking (48\%), tobacco using (8\%) and betel nuts chewing (28\%) influenced gingivitis. Most of the respondents (89\%) were suffering from gingivitis for at least one year and gum bleeding was documented in 99\% of respondents. Conclusion: Dietary and behavioral patterns were found to be responsible for gingivitis.
\end{abstract}

Keywords: Gingivitis; determinants.

Delta Med Col J. Jan 2015;3(1):9-12

\section{Introduction}

Gingivitis may be defined as inflammation of the gums (gingiva). It commonly occurs because of films of bacteria that accumulate on the teeth plaque; this type is called plaque-induced gingivitis. Gingivitis is a non-destructive type of periodontal disease. If left untreated, gingivitis can progress to periodontitis, which is more serious and can eventually lead to loss of teeth. A patient with gingivitis will have red and puffy gums, and they will most likely bleed when they brush their teeth. Generally, gingivitis resolves with good oral hygiene - frequent brushing, as well as dental flossing. Some people find that using of an antiseptic mouthwash, along with proper brushing of teeth and flossing also help. In mild cases of gingivitis, patients may not even know they have it, because symptoms are mild. However, the condition should be taken seriously and addressed immediately. ${ }^{1}$ A study was done to see factors affecting oral malodor in periodontitis and

1. Senior Lecturer, Dept. of Public Health, Atish Dipanker University of Science and Technology, Dhaka, Bangladesh.

2. Fellow, Dept. of Community Nutrition, Faculty of Public Health, Bangladesh University of Health Sciences (BUHS), Dhaka, Bangladesh.

3. Associate Professor, Dept. of Public Health, Atish Dipanker University of Science and Technology, Dhaka, Bangladesh.

Correspondence: Md. Monoarul Haque. e-mail: monoarmunna@yahoo.com 
gingivitis patients and showed that in the gingivitis group, oral malodor was significantly correlated with the plaque index, bleeding on probing, and tongue coating. ${ }^{2}$ In developed countries, this disease occurs mostly in young adults. In developing countries, necrotizing ulcerative gingivitis (NUG) may occur in children of low socioeconomic group, usually occurring with malnutrition, especially inadequate protein intake and shortly after the onset of viral infections (e.g. measles). ${ }^{3}$ Predisposing factors include smoking, viral respiratory infections and immune deficiency disorders such as in HIV/AIDS. Uncommonly, except in lower socioeconomic group, this typically affects adolescents and young adults, especially in institutions, armed forces, etc., or people suffering from HIV/AIDS. ${ }^{4}$ The disease has occurred in epidemic-like patterns, but it is not contagious. $^{3}$ The objective of the current study was to find out the factors affecting gingivitis in Bangladesh perspective.

\section{Materials and method}

A cross sectional study was conducted in the outpatient department in Bangabndhu Sheikh Mujib Medical University (BSMMU), Dhaka, Bangladesh among 200 patients aged between 15-40 years from 01 May to 31 August, 2008. Non probability purposive sampling method was used for data collection. Mentally retarded and insane people were excluded from the study. The socio-economic classification in this study was made according to 2006 Gross National Income (GNI) per capita and using the calculation of World Bank (WB). ${ }^{5}$ The groups were: low-income $\$ 75.41$ or less (BDT $\leq 5360)$, lower middle-income \$75.5-\$299.58 (BDT 5361-21270), upper middle-income \$299.68\$926.25 (BDT 21271-65761) and high-income $\$ 926.33$ or more (BDT $\geq 65762$ ). Before data collection, consent was taken from all the patients. Data were collected using pretested semi structured questionnaire by face to face interview and examining teeth including gum diameter and supragingival calculus or plaque and depth of periodontal pocket. After data collection, data were sorted, scrutinized based on the selection criteria and then data were analyzed by calculator and by SPSS version 12.0 programme.

\section{Results}

Regarding educational status of the study subjects, illiterate, primary, secondary, higher secondary education and above were $18.5 \%, 27 \%, 28 \%$ and $26.5 \%$ respectively. About $45 \%$ were housewife. Male and female were almost equal in numbers. About $75 \%$ came from lower income group. Most of them (93\%) were Muslim (Table I).

Most of the respondents (89\%) were suffering from gingivitis for at least one year and did not go for any type of dental check up. About $69 \%$ of respondents brushed their teeth with tooth paste and $85 \%$ of them did not use any type of mouth wash. Almost all of them presented with gum bleeding, calculus and plaque. Twenty eight percent respondents had periodontal pocket (Table II).

Table I: Socio-demographic characteristics of respondents $(\mathrm{N}=\mathbf{2 0 0})$

\begin{tabular}{lcc}
\hline Variables & Frequency & Percentage \\
\hline Education & & \\
$\quad$ No schooling/illiterate & 37 & 18.5 \\
$\quad$ Primary & 54 & 27 \\
$\quad$ Secondary & 56 & 28 \\
$\quad$ Higher secondary \& & 53 & 26.5 \\
$\quad$ above & & \\
Occupation & & \\
$\quad$ Housewife & 90 & 45 \\
$\quad$ Business & 33 & 16.5 \\
$\quad$ Farmer & 17 & 8.5 \\
$\quad$ Rickshaw puller & 27 & 13.5 \\
$\quad$ Others & 33 & 16.5 \\
Sex & & \\
$\quad$ Male & 104 & 52 \\
$\quad$ Female & 96 & 48 \\
Monthly income(BDT) & & \\
Lower income (<5360) & 149 & 74.5 \\
Middle income (5360-21270) & 39 & 19.5 \\
Upper middle-high income (21271-65761) & 12 & 6 \\
Religion & & \\
Muslim & 186 & 93 \\
Hindu & 9 & 4.5 \\
Christian & 5 & 2.5 \\
\hline
\end{tabular}


Smoking, tobacco intake and betel nuts chewing were reported in $48 \%, 8 \%$ and $28 \%$ of respondents respectively. Among dietary factors, sweet (80\%) and fast food (14\%) were mostly responsible for gingivitis (Table III).

Table II: Pattern of gingivitis of respondents $(\mathrm{N}=\mathbf{2 0 0})$

\begin{tabular}{|c|c|c|}
\hline Variables & Frequency & Percentage \\
\hline \multicolumn{3}{|l|}{ Duration of suffering } \\
\hline 0-12 month & 178 & 89 \\
\hline 13-24 month & 16 & 8 \\
\hline$\geq 25$ month & 6 & 3 \\
\hline \multicolumn{3}{|l|}{ Dental check up } \\
\hline Yes & 7 & 3.5 \\
\hline No & 193 & 96.5 \\
\hline \multicolumn{3}{|l|}{ Brushing activity } \\
\hline Brushing with tooth paste & 138 & 69 \\
\hline Using hand with tooth paste & 18 & 9 \\
\hline Using hand with different items & 44 & 22 \\
\hline \multicolumn{3}{|l|}{ Interdental brushing } \\
\hline Yes & 14 & 7 \\
\hline No & 186 & 93 \\
\hline \multicolumn{3}{|l|}{ Mouth wash } \\
\hline Yes & 30 & 15 \\
\hline No & 170 & 85 \\
\hline \multicolumn{3}{|l|}{ Gum bleeding } \\
\hline Present & 198 & 99 \\
\hline Absent & 2 & 1 \\
\hline \multicolumn{3}{|l|}{ Supragingival calculus } \\
\hline Present & 197 & 98.5 \\
\hline Absent & 3 & 1.5 \\
\hline \multicolumn{3}{|l|}{ Subgingival calculus } \\
\hline Present & 198 & 99 \\
\hline Absent & 2 & 1 \\
\hline \multicolumn{3}{|l|}{ Supragingival plaque } \\
\hline Present & 196 & 98 \\
\hline Absent & 4 & 2 \\
\hline \multicolumn{3}{|l|}{ Subgingival plaque } \\
\hline Present & 197 & 98.5 \\
\hline Absent & 3 & 1.5 \\
\hline \multicolumn{3}{|l|}{ Periodontal pocket depth } \\
\hline Present & 56 & 28 \\
\hline Absent & 144 & 72 \\
\hline
\end{tabular}

Table III: Distribution factors affecting gingivitis of respondents $(\mathrm{N}=\mathbf{2 0 0})$

\begin{tabular}{lcc}
\hline Variables & Frequency & Percentage \\
\hline Habit & & \\
Smoking & 95 & 48 \\
Tobacco using & 16 & 8.1 \\
Betel nuts chewing & 56 & 28.3 \\
Others & 31 & 15.7 \\
Dietary factors & & \\
Sweet & 160 & 80 \\
Chocolate \& chewing gum & 2 & 1 \\
Fast food & 28 & 14 \\
Others & 10 & 5 \\
\hline
\end{tabular}

\section{Discussion}

This study provided some important information about gingivitis. The present study revealed that $48 \%, 8 \%$ and $28 \%$ of respondents had habits of smoking, tobacco using and betel nuts chewing respectively. Among dietary factors, sweet (80\%) and fast food (14\%) were mostly responsible for gingivitis. Some evidence showed that smoking is the single major preventable risk factor for periodontal disease. Smoking can cause bone loss and gum recession even in the absence of periodontal disease. The risk of periodontal disease increases with the number of cigarettes smoked per day. Smoking cigars and pipes carries the same risks as smoking cigarettes. It is also believed that chewing tobacco plays an aetiological role in gingivitis. Long-term abuse of alcohol and certain types of illegal drugs (amphetamines) can damage gums and teeth. A healthy diet, including eating fruits and vegetables rich in vitamin $\mathrm{C}$, is important for good oral health. Malnutrition is a risk factor for periodontal disease. ${ }^{6,7}$

The present study found that about $69 \%$ of respondents brushed their tooth with tooth paste and $85 \%$ of them did not use any type of mouth wash. It was found that most of the patients (89\%) were suffering from gingivitis for at least one year 
and they did not go for dental check up. Almost all of them presented with gum bleeding, calculus, plaque and $28 \%$ had periodontal pocket. Lack of oral hygiene, such as not brushing of teeth or flossing regularly, encourages bacterial buildup and plaque formation. ${ }^{6}$ Evidence supported that pathological factors include bone resorption as a sequel to microbial induced periodontal diseases. In addition to psychological factors, various forms of trauma - such as vigorous brushing of teeth, aberrant frenal attachment, occlusal injury, operative procedures have been thought to play a role in the aetiology of gingivitis. $^{7}$ Epidemiological studies have concluded that traumatic tooth brushing may be associated with gingivitis. ${ }^{8}$ Faculty of Dentistry of University of Toronto encouraged brushing teeth at least twice a day and flossing at least once a day. The most important time to brush is before going to bed. This is because while sleeping there is less saliva in mouth and less frequent swallowing, so there is less saliva available to wash away the bacteria. It is also important that one does not eat after brushing teeth, especially not any foods that are sticky or sugary. ${ }^{9}$ There is a misconception that eating certain foods can help keep mouth clean. In fact, there are no foods that clean teeth. One can accomplish this only through brushing, flossing and other means of mechanical cleaning. Some foods can even increase plaque build-up. Examples of such foods are sticky candies, soft, mushy foods like breads and pasta, as well as sugary foods and sweetened drinks. If one does eat sweets, eat them all at one time rather than throughout the day, and brush teeth right afterwards. ${ }^{9}$

This study provided a vivid picture of factors affecting gingivitis among patients aged 15 to 40 years in Bangladesh. Diet and behavior were responsible for gingivitis. The study result should not be generalized and needs further large scale research.
Acknowledgment: The authors express their sincere thanks to all the patients of this study. No external funding was provided for this study.

\section{References}

1. Hawranko BL. 1999 World Workshop in Clinical Periodontics [Internet]. [cited 2014 Dec 10]. Available from: http://www.medicalnewstoday.com /articles/241721.php.

2. Pham TA, Ueno M, Shinada K, Kawaguchi Y. Factors Affecting Oral Malodor in Periodontitis and Gingivitis Patients. J Investig Clin Dent. 2012;3(4):284-90.

3. Lindhe J, Lang NP, Karring T, editors. Clinical Periodontology and Implant Dentistry. 5th ed. Copenhagen: Blackwell Munksgaard; 2008.

4. Scully C. Oral and Maxillofacial Medicine: The Basis of Diagnosis and Treatment. 2nd ed. Edinburgh: Churchill Livingstone; 2008.

5. Haque ANMN. The Middle-Income Matrix. The Daily Star. 2007 Nov 18. [cited 2014 Dec 10]. Available from: http://www.wsulibs.wsu.edu/ quickguides/nlm.

6. ADAM [Internet]. Gingivitis - Overview. The New York Times. [Reviewed 2013 Nov 3; cited 2014 Dec 10]. Available from: http://www.nytimes.com /health/guides/disease /gingivitis/risk-factors.html.

7. Amran AG, Ataa MAS. Statistical Analysis of the Prevalence, Severity and Some Possible Etiologic Factors of Gingivitis among the Adult Population of Thamar City, Yemen. RSBO. 2011;8(3):305-13

8. Addy M, Mostafa P, Newcombe RG. Dentine Hypersensitivity: The Distribution of Recession, Sensitivity and Plaque. J Dent. 1987;15:242-48.

9. Sangnes G, Gjermo P. Prevalence of Oral Soft and Hard Tissue Lesions Related to Mechanical Tooth Cleansing Procedures. Community Dent Oral Epidemiol. 1976;4(2):77-83. 